\title{
Coronavirus Pandemic What Are We Doing
}

\author{
Jose Carlos Herrera Garcia* \\ Pneumology/Respiratory Unit, Hospital Angeles Puebla, Mexico \\ *Corresponding author: Jose Carlos Herrera Garcia, Pneumology/Respiratory Unit, Hospital Angeles Puebla, Mexico.
}

To Cite This Article: Jose Carlos Herrera Garcia, Coronavirus Pandemic What Are We Doing. 2020 - 9(6). AJBSR.MS.ID.001450.

DOI: 10.34297/AJBSR.2020.09.001450.

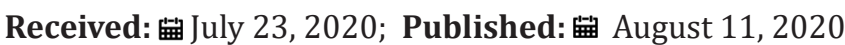

\section{Short Communication}

Since the discovery of the new pneumonia due to coronavirus in wuhan and spreading throughout the world, it has made us reflect on the state of the human being in this world, the lethality of this virus has shown us how fragile we are in the face of biological challenges, this a pandemic with more than 600,000 deaths describes the need to think about the life we lead and the way we lead it, to be on multiple treatments and to see the mortality of patients with respiratory distress who go to the emergency services in each corner of the world is to see how the pathophysiology of the virus reminds us of clot formation and rupture of the capillary alveolar membrane capable of producing severe hypoxemia that lead the patient to a state of multisystemic shock or cytokine storm that increase patient mortality.

For more than 5 months fighting against this adversity, it is important to stop to think about all the information that has not been left during this time, where the multiverse treatment coupled with an immune state of the patient that will allow him to get ahead of the disease, clear effects we have on treatment such as azithromycin treatment described by lancet as a drug capable of inhibiting the activity of IL-6 and TNF, important factors in the development of the disease, antipyretics such as paracetamol that only decrease the symptoms of patients, anticoagulation serious treatment that has allowed patients to improve, already with explicit work on the formation of clots and recently the findings of micro thrombi in the entire pulmonary and systemic vasculature that have not allowed the use of direct anticoagulants such as rivaroxaban and apixaban with excellent responses Given the lack of enoxaparin in some populations, oxygen is are pulsive in the disease that allows patients to recover due to the improvement of the diffusion of gases in the membrane and recovery of the oxemia, being this factor to definitely give the go-ahead to the fifth vital sign that is the oximetry, never taken into account like in this pandemic.

During the treatment of patients, we know that the immune response plays a fundamental role in the inhibition of the disease, but when we are faced with hyperstimulatory or hyperinflammatory responses, we must help ourselves with biological drugs that inhibit the response of cytokines, such as tocilizumab and ruloxitinib that so far, we have described in a series of cases the favorable response to these treatments, of course as long as it is indicated in the hyperstimulating response of the disease. During the pandemic and seeing patients with different comorbidities, it is worth mentioning that hypertension is the most important risk factor for severe pneumonia, but not for patients with autoimmune diseases, who in a certain way have seen them as protected by the treatments they carry out. it does not allow an exaggerated response to inflammation. Other related factors such as: overweight, obesity, diabetes, hypothyroidism, cancer, renal failure and COPD etc. are comorbidities that substantially increase mortality.

After describing the treatments and vision of patients with coronavirus, it is important to highlight that sanitary measures and a face mask are the cheapest weapons to stop the spread of the virus in any circumstance where we are not involved, taking into account that the same society has than taking the helm and being empathetic to vulnerable groups. Regarding diagnosis, the PCR test remains the gold standard, so far, the only one supported to determine coronavirus infection, antibodies and rapid tests not yet ready to be taken into account as diagnoses, perhaps for followups or procedures to acquire antibodies, but we know that they are not a reliable source in the diagnosis. I have nothing left but to continue in the fight, with the fear of all together, with the need to 
end this storm of patients who continue to fight day by day, as well as a health system that is not seen for when I can get up and give finally to this pandemic.

\section{Acknowledgment}

None.

\section{Conflict of Interest}

No conflict of interest. 